\title{
Susceptibility of Beta-Haemolytic Escherichia coli to Commonly Used Antibiotics in Selected Hospitals in Delta State, Southern Nigeria
}

\author{
Itohan S Aghemwenhio ${ }^{1}$, Adesoji A Timilehin ${ }^{1}$ and Alpheus GA ${ }^{2}$
}

${ }^{1}$ Biological Sciences Department, Federal University, Dutsin-ma, Nigeria

${ }^{2}$ Department of Microbiology, Delta state university, Abraka

Corresponding author: Itohan S Aghemwenhio, Biological Sciences Department, Federal University, Dutsin-ma, Nigeria, Tel: +2349027260803; Email: itohansandra@yahoo.com

Received Date: February 10, 2017; Accepted Date: March 13, 2017; Published Date: March 20, 2017

Copyright: (c) 2017 Aghemwenhio S, et al. This is an open-access article distributed under the terms of the Creative Commons Attribution License, which permits unrestricted use, distribution, and reproduction in any medium, provided the original author and source are credited.

Citation: Aghemwenhio IS, Timilehin AA, Alpheus GA. Susceptibility of Beta-Haemolytic Eschericia Coli to Commonly Used Antibiotics in Selected Hospitals in Delta State, Southern Nigeria. Arch Clin Microbiol. 2017, 8:2.

\section{Abstract}

Antimicrobial resistance in the treatment of urinary tract infections is a major health problem. This study evaluates the pattern of susceptibility of pathogens commonly responsible for urinary tract infections (UTIs) to commonly used antimicrobial agents. Midstream urine samples of 80 patients ( 30 males and 50 females) and 20 diarrhea samples from 10 males and 10 females, who were attending clinics in Eku, Sapele and Abraka general hospitals, Delta state, between September and October 2007 were examined. Susceptibility of the urine and diarrhea bacteria isolates to ten commonly used antibiotics were investigated. Thirty five Escherichia coli isolates were obtained from the urine samples, out of which were nine hemolytic strains (25.7\%), and $10 \mathrm{E}$. coli isolates from diarrhea, out of which was one strain (10\%). All the hemolytic strains exhibited a significantly high resistance to septrin, sparfloxacin, amoxicillin, streptomycin, chloramphenicol but were either moderately or highly sensitive to the augmentin and gentamycin. I conclude that the incidence of hemolytic strains in urinary tract infection was higher than that observed from diarrhea because Escherichia coli strains that cause urinary tract infection typically produce hemolysins which is an important virulent factorin pathogenesis of infection, whereas those strains that are part of the gastro intestinal micro flora may not produce hemolysins and majority of the antimicrobial agents that are commonly used to treat UTIs caused by Escherichia coli in the hospitals are no longer effective due to hemolysin production. Therefore, the development and strict management of antimicrobial policy, and surveillance for resistant organisms should be given priority in Nigeria.

Keywords: Urinary tract infections; Antimicrobial agents; Bacterial isolates; Hemolysin

\section{Introduction}

The field of Microbiology helps to distinguish beneficial microorganisms from harmful ones. Some microorganisms live in mutual co-existence with humans as normal flora of the body and these microorganisms may be pathogenic when they become opportunistic. Eschericia coli one of such opportunistic microorganisms is a known member of the family Enterobacteriaceae. It is a gram negative non spore forming, motile, facultative anaerobic, short rod measuring 0.3-1.5 $\mu \mathrm{m}$ in diameter. Usually the genus Eshericia contains five species; Eschericia coli, E. fergusonii, E. plattae, E. vulneris and E. hermanii. E. coli however is the most predominant, facultative organism found in the large bowels of humans and is the most frequently isolated enteric Bacillus in the clinical laboratory. It is unique among other opportunistic pathogens in that it has been associated with human gastro intestinal diseases particularly in children and travelers to developing nations.

Eschericia coli are grouped into enterotoxigenic, enteroinvasive, enteropathogenic and enterohaemorrhagic $E$. coli [1]. E. coli has been the subject of more experimental research than any other microorganism especially in the field of molecular genetics. $E$. coli is highly ubiquitous, hence it's frequently studied in Microbiology and is the current "workhouse" in molecular biology, its structure is clear and makes for an excellent target for novice and advanced students of the life sciences. It has also been implicated as causative agents of various infections in man and animals such as: Urinary tract infections; cystitis and pyelonephritis, pneumonia, bacteremia and meningitis. The ability to produce beta hemolysin is thought to be one of the virulence factors of pathogenic $E$. coli . Two kinds of hemolysins are produced. One designated alpha-hemolysin is soluble hemolysin found in cellfree culture supernatant. It occurs in two molecular species, which differ in size, it is sensitive to trypsin and requires calcium ion for activation. The synthesis of alpha-hemolysin is controlled by transmissible plasmid. It is not toxic in animals but its invitro hemolytic activity is neutralized by homologous antibody. The other, Beta-hemolysin is a cell bound hemolysin. Both lyze a wide variety of erythrocytes. These hemolysins do not appear to be associated with pathogenesis of infection with hemolytic strains [2]. Cytotoxicity to phagocytes, which is mediated by or closely linked genetically alpha hemolysin may be a mechanism by which alpha hemolytic strains of $E$. coli strengthen their 
ability to maintain and establish

infection [3]. The effect of osmolarity, temperature and anaerobiosis increase hemolysin production in hemolytic strains isolated from UTI. The haemolysin production, haemagglutinating activity with human O group erythrocyte and antibiotic susceptibility of 130 uropathogenic $E$. coli strains were studied. $43 \%$ of the strains produced hemolysins and $39 \%$ showed haemagglutination activity. $12 \%$ of the haemagglutinating strains were inhibited by D-mannose. $45 \%$ of the hemolytic strains showed haemagglutinating activity. $85 \%$ of the $130 \mathrm{E}$. coli strains were found to be multiple resistant to antibiotics [4]. Urinary tract infections (UTIs) are common type of bacterial infection accounting for reasonably high health care expenditures in people of all ages, with more than 35 million medically treated infections each year [5]. Young women, the elderly and those undergoing genitourinary instrumentation or catheterization, among others, are also at risk [6]. UTIs are the leading cause of gram-negative bacteremia. UTIs are usually treated with antibiotics including nalidixic acid, nitrofurantoin, ofloxacin, perfloxacin, ciprofloxacin, gentamycin, etc. In the last couple of years, there has been a lot of focus in scientific literature on inappropriate use of antimicrobial agents and the spread of bacterial resistance. The widespread and inappropriate use of antibiotics is recognized as a significant contributing factor to the spread of bacterial resistance and the development of resistance to antimicrobial agents. The emergence of antimicrobial resistance in the management of urinary tract infections is an important public health issue. While many antibiotics including penicillin, macrolides and tetracycline were very useful in the treatment of urinary tract infections in the past, the rates of bacterial resistance to antimicrobial agents has significantly increased and are increasing in many countries in recent times [7]. Despite the well-publicized concerns about the problems of inappropriate use of antimicrobial agents, or use of broad spectrum antibiotics (when narrow spectrum drugs would be effective), increasing resistance of bacteria causing urinary tract infections to antimicrobial agents remains a serious problem. With the high prevalence of fake and substandard drugs available in Nigeria [8], the rate of bacterial resistance antimicrobial agents in the management of UTIs is likely to be much higher than many other countries. This study therefore seeks to highlight the degree of susceptibility of $E$. coli to the commonly used antibiotics in Eku, Sapele and Abraka areas of Delta state. The result of this, study is expected to provide useful information which would assist physicians in their good 2 prescribing habits towards a better management of urinary tract infections.

\section{Material and Methods}

\section{Study area, samples collection and bacteria isolation}

Samples from General hospital, Sapele and Eku general hospital in Delta State, Southern Nigeria were selected for this study. Mid-stream urine and stool samples from diarrhea patient were collected from 100 patients, 60 males and 40 females attending the clinic. (family practice clinic, consultant outpatient clinic, obstetrics and gynecology clinic, and accident and emergency clinic)from these hospitals. Urine samples $(n=50)$ and stool samples $(n=10)$ were collected from Sapele hospital while 30 and 10 urine and stool samples respectively were collected from Eku hospital. Samples were collected using sterile McCartney bottles. Urine samples and 10 diarrhea samples were collected from Sapele hospital and 30 urine and 10 diarrhea samples from Eku hospital. The specimens were collected by voiding the first part of the urination into toilet bowl, before collecting the mid-stream portion. Then afterwards, samples were taken to the laboratory for analysis, stored at $4^{\circ} \mathrm{C}$ and cultured within 24 hours. Both urine and stool samples were streaked using sterile inoculating loop on sterile MacConkey agar in petri dishes. They were thereafter, incubated at $37^{\circ} \mathrm{C}$ for 18-24 hours in an incubator. Lactose fermenters showed distinct circular edge colonies with smooth surface with a rose pigmentation while non-lactose fermenters are recognized by their colorless circular colonies. Lactose fermenters were suspected as They were, therefore, re-isolated by streaking out on nutrient agar. Pure colonies were later gram stained, colonies showing Gram-negative short rods were thereafter subcultured into Eosin methylene blue agar (EMB) and for on-ward transfer into Nutrient agar slants and stored at $4^{\circ} \mathrm{C}$ in a refrigerator. Stool samples were collected from twenty patients from the wards with MacCartney bottles. These containers were highly secured and transferred directly to the laboratory where they were immediately plated out in an appropriate media.

\section{Biochemical characterization and Identification of bacteria Isolates}

Isolated $E$. coli were characterized on the based on cultural appearance, colony morphology and conventional biochemical tests such as urease test, citrate utilization test, catalase test, methyl red test, haemolysis on blood agar, indole test according to the method of Olutiola et al. based on Bergey's manual of systemic nomenclature (2007).

\section{Heamolysis test for $E_{0}$ coli}

A loopful of confirmed $E$. coli isolates were streaked on blood agar and thereafter incubated at $37^{\circ} \mathrm{C}$ for 24 hours. Isolates on plates were therefore observed for clear zone round the culture. Clear zone round the isolates signifies beta hemolytic activity.

\section{Inoculation}

Each urine sample was inoculated by dipping into universal container containing urine a flamed inoculating loop. This was used to make a pool on the medium (MacConkey and streak, after reflaming inoculating loop. These lactose fermenters have distinct circular edge colonies, which are smooth surface with rose pink pigmentation. Non-lactose fermenters also grow on the medium but are recognized by their colorless circular colonies. Suspected lactose fermenting $E$. coli strains gotten after 24 hours incubated at $37^{\circ} \mathrm{C}$ were subjected to gram staining. Colonies 2 showing Gram-negative short bacilli were subcultured into Eosin methylene blue agar and into 3 Nutrient agar slants and stored at $4^{\circ} \mathrm{C}$ in a refrigerator. They were later used for biochemical and 4 sensitivity test. Stool samples were inoculated on already solidified and dried MacConkey agar 5 plates with sterile inoculating loop. The plates were incubated at 
$37^{\circ} \mathrm{C}$ for $18-24$ hours, only 6 plates showing distinct growth of lactose fermenting colonies were picked from each plate and inoculated into MacConkey medium for purification.

\section{Identification of microorganisms}

Identification of isolates was done using Bergey's manual of determinative Bacteriology. Organisms were identified and characterized on basis of cultural appearance, colony morphology and conventional biochemical tests.

Cultural characteristics: Characteristics like shape, margin, elevation, size etc were noted on plates of nutrient agar and MacConkey agar.

Haemolysis on blood agar: Small inocula of suspected isolates of $E$. coli were streaked on blood agar (Nutrient agar plus sterile blood) plates and incubated for 24 hours at $37^{\circ} \mathrm{C}$. Plates were observed for beta hemolysis i.e. those with clear zones.

\section{Antibiotics sensitivity testing}

Preparation of agar: Muller-Hinton agar was autoclaved, allowed to cool, the freshly prepared medium was poured in Petri dishes and stored in refrigerator at $80 \mathrm{C}$, a batch of plates were examined for sterility by incubating at $35^{\circ} \mathrm{C}$ for 24 hours.

Turbidity standard was used to standardize inoculums density for susceptibility test, a $\mathrm{BaSO}_{4} 0.5 \mathrm{McF}$ (arland standard was prepared as follows; a $0.5 \mathrm{ml}$ aliquot of $0.048 \mathrm{~mol} / \mathrm{L} \mathrm{BaCl}_{2}$ $\left(1.175 \% \mathrm{w} / \mathrm{v} \mathrm{BaCl}\right.$. $2 \mathrm{H}_{2} \mathrm{O}$ ) was added to $99.5 \mathrm{ml}$ of $0.18 \mathrm{~mol} / \mathrm{ml}$ $\mathrm{H}_{2} \mathrm{SO}_{4}(1 \% \mathrm{v} / \mathrm{v})$ with constant stirring to maintain a suspension, the correct turbidity standard was verified using a spectrophotometer with a $1 \mathrm{~cm}$ light path and matched cuvette to determine the absorbance. The absorbance at $625 \mathrm{~nm}$ was 0.0008 to 0.10 for McFarland standard. Barium sulphate suspension was transferred in $4 \mathrm{ml}$ aliquots into screw caps tubes of same size as those used in growing the inoculums, tubes were tightly sealed and stored in dark room at room temperature.

Inoculum preparation: Inoculum was prepared by making a direct broth of isolated colonies selected from 18-24 hours agar plates, suspension was adjusted to match $0.5 \mathrm{ml}$ McFarland standard, using sterile saline. Inoculation of test plates was done within 15 minutes after adjusting turbidity of the inoculums suspension, a sterile cotton swab was dipped in to adjusted suspension, dried surface of agar plates was inoculated by streaking swab over the entire sterile agar surface, the lid was left ajar for 3-5 minutes to allow excess surface moisture to be absorbed.

The sensitivity of $E$. coli isolates to 10 different antibiotics was determined by using Kirby Bauer disc diffusion method, antibiotics susceptibility disc containing the following antibiotics augmentin $(30 \mu \mathrm{g})$, gentamycin $(10 \mu \mathrm{g})$, tarivid $(10 \mu \mathrm{g})$, pefloxacin $(10 \mu \mathrm{g})$, streptomycin $(20 \mu \mathrm{g})$, septrin $(30 \mu \mathrm{g})$, chloramphenicol $(30 \mu \mathrm{g})$, sparfloxacin $(10 \mu \mathrm{g})$, ciprofloxacin $(10$ $\mu \mathrm{g})$, amoxicillin $(30 \mu \mathrm{g})$. The plates were incubated for 24 hours at $37^{\circ} \mathrm{C}$. According to $\mathrm{CLSI}$ recommendation (M31-A3) E. coli
ATCC 25922 was used as control strain. Clear zones of inhibition surrounding the antibiotics disk were, thereafter, measured with a ruler. Results were then compared with Clinical and Laboratory Standard Unit [9] to determine resistant and sensitivity strains.

\section{Results}

A total of 100 samples (urine, $n=80$ and Stool, $n=20$ ) were collected for this study while $45 \mathrm{E}$. coli (urine, $\mathrm{n}=35$ and stool, $n=10$ ) were isolated from each samples from selected hospital. Out 4 of thirty samples, only one showed these characteristics (Tables 1 and 2).

Table 1 Number of samples, E. coli isolates and haemolytic strains from urine and stool.

\begin{tabular}{|l|l|l|l|l|}
\hline Sample & $\begin{array}{l}\text { Total no. of } \\
\text { samples }\end{array}$ & $\begin{array}{l}\text { No. of E. coli } \\
\text { isolates }\end{array}$ & $\begin{array}{l}\text { No. of } \\
\text { hemolytic } \\
\text { strains }\end{array}$ & $\begin{array}{l}\text { Stains } \\
\text { percentage }\end{array}$ \\
\hline Urine & 80 & 35 & 9 & 25.7 \\
\hline Stool & 20 & 10 & 1 & 10 \\
\hline
\end{tabular}

\section{Discussion}

Result from the work reveals that the incidence of haemolytic strains of $E$. coli is higher in urinary tract infections than in diarrhea of the samples screened; this is because $E$. coli strains that because infections typically produce hemolysins,

whereas those strains that are part of the gastrointestinal flora may not produce hemolysins. [1] Haemolysin production may be an important virulent factor in pathogenesis of urinary tract infection. There is a gene responsible for the coding of cytotoxic factor in hemolytic strains of $E$. coli [10]. The antibiotics used in this study are some of the antimicrobial drugs commonly used to treat UTI in Southern Nigeria.

The hemolytic strains were highly resistant to septrin $(80 \%)$, sparfloxacin (60\%) and moxicillin (50\%). The high level of resistance observed for these drugs could be attributed to their gross misuse by both clinicians and non-clinicians alike. This condition could be aggravated by the relative cheapness, convenience of administration, since they are available in tablets and capsules. Ignorance as regards the implications of drugs resistance and ineffective laws controlling the scale and use of antibiotics are possible additional reasons for misuse, also Rfactor mediated drug resistance poses a threat to successful treatment of Gram negative infections with antibiotics [11].

The most useful drug for the treatment of $E$. coli infection in urinary tract infection and diarrhea as obtained from the antibiotic sensitivity test of all the isolates is augmentin, since it was observed from this study that all haemolytic strains $E$. coli isolates were sensitive to augmentin, (100\%), this therefore, could be the most useful drug for its treatment. The strains were sensitive to augmentin because augmentin is a combination of 
clavulanic acid and penicillin, hence drug combination therapy may prevent the emergence of resistance organisms [12].

Table 2 Antibiotic sensitivity and resistance percentage of $E$. coli haemolytic.

\begin{tabular}{|c|c|c|c|c|}
\hline Antibiotic & Number of sensitive isolates & $\begin{array}{l}\text { Percentage }(\%) \quad \text { of } \\
\text { sensitivity }\end{array}$ & Number of resistant isolates & $\begin{array}{l}\text { Percentage }(\%) \\
\text { resistant isolates }\end{array}$ of \\
\hline Augmentin & 10 & 100 & 0 & 0 \\
\hline Gentamycin & 8 & 80 & 2 & 20 \\
\hline Pefloxacin & 8 & 80 & 2 & 20 \\
\hline Tarivid & 8 & 80 & 2 & 20 \\
\hline Streptomycin & 6 & 60 & 4 & 40 \\
\hline Septin & 2 & 20 & 8 & 80 \\
\hline Chloramphenicol & 6 & 60 & 4 & 40 \\
\hline Sparfloxacin & 4 & 40 & 6 & 60 \\
\hline Ciprofloxacin & 8 & 80 & 2 & 20 \\
\hline Amoxicillin & 5 & 50 & 5 & 50 \\
\hline
\end{tabular}

\section{Conclusion}

This work revealed that $E$. coli strains that cause urinary tract infection typically produce hemolysins [13]. Haemolysin production may therefore be important in the pathogenesis of urinary tract infection and the hemolytic strains were highly resistant to antibiotic drugs, I therefore recommend that Research effort be directed at the exact role of haemolysin, as a proper understanding of the virulence factors will help in developing adequate preventive measures, there is the need for proper awareness on the part of the general public to know the dangers of abusing antibiotics, I also recommend the development of antimicrobial policy (which is currently nonexistence in Nigeria). Strict management of such antibiotic policies and surveillance programs for resistant organisms, together with infection control procedures, need to be implemented and continuously audited as suggested by Elliott and Lambert [14].

\section{References}

1. Geo FB, Janet SB, Stephen AM (2004) Medical Microbiology (18th Edn). Prentice hall, Newyork, USA pp 411.

2. Freeman BA (1991) Burrow's Textbook of Microbiology. (11thEdn) WB Saunders company Philadelphia. pp 1086.

3. Gadeberg OV, Orskov I (1984) In vitro cytotoxic effect of haemolytic Eschericia coli on human blood granulocytes. Annual Rev Microbiol 14: 471-485.

4. Coser G (1991) Antibiotics susceptibility, haemolysin production and hem-agglutinating activity of Uropathogenic Eschericia coli. J Med Microbiol 35: 303-307.
5. Mindbranch Inc (2004) Stakeholder Opinions: Urinary Tract Infections -Ciprofloxacin Leads the Way. Data monitor, USA.

6. Kunin CM (1994) Urinary tract infections in females. Clin Infect Dis18: 1-12.

7. Ho P, Que T, Chiu SS, Yung RWH, Tsang DNC, et al. (2004) Fluoroquinolone and other antimicrobial resistance invasive pneumococci, HongKong, 1995-2001. Emerg Infect Dis10: 1250-1257.

8. Raufu A (2002) Influx of fake drugs to Nigeria worries health experts. BMJ 324: 698.

9. Clinical and Laboratory Standard Institute (2008): Performance Standards for antimicrobial disc susceptibility test; approved standard-Tenth edition.

10. Caprioli A, Falbo V, Ruggeri FM, Baldassari L, Ippolito G, et al. (1997) Cytotoxic necrotizing factor production by hemolytic strains of Eschericia coli causing extra intestinal infections. J clin microbiol 25: 146-149.

11. Prescott LM, Harley JP, Klien DA (2005) Microbiology (6th edn). McGraw Hill Company, pp 992.

12. Matsen JM, Barry AL (1974) Manual of Clinical Microbiology. (2nd edn). America Society for Microbiology, Washington DC, USA pp 418-427.

13. Onanuga A, Oyi AR, Olayinka BO, Onaolapo JA (2005) Prevalence of community-associated multi-resistant Staphylococcus aureus among healthy women in Abuja, Nigeria. Afr J Biotech 4: 942-945.

14. Elliott TS, Lambert PA (1999) Antimicrobial resistance in 13 mechanisms and management British Medical Bulletin. 55: 259-276. 